Available online at www.sciencedirect.com

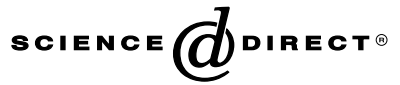

\title{
False memories and delusional ideation in normal healthy subjects
}

\author{
Keith R. Laws *, Reena Bhatt \\ Brain and Cognition Research Group, Division of Psychology, Nottingham Trent University, Burton Street, \\ Nottingham NGI 4BU, UK
}

Received 20 July 2004; accepted 7 March 2005

\begin{abstract}
Studies have reported substantial mnestic deficits in patients with schizophrenia. Most of this research, however, has focussed on errors of omission (poor recall/recognition) rather than commission (such as false recall/recognition). Nevertheless, recent studies report that schizophrenics show increased false recognition and speculate that this may underpin delusional ideation (Moritz et al., 2004). No previous study has examined whether such memory problems exist in normal individuals who may be prone to delusional thinking. Using the Roediger and McDermott (1995) paradigm, we investigated memory functioning in 105 normal healthy subjects divided according to performance on a measure of delusional ideation (Peters et al. Delusional Inventory: PDI Peters et al., 1999). We found significantly poorer recall in the high than low PDI group. Moreover, high PDI scorers also made more false-alarm memory recalls than low PDI scorers. In a recognition test, high and low PDI subjects did not differ in the confidence they attached to recognition of studied items, but high PDI subjects gave greater confidence for falsely accepting unseen items. This suggests that healthy subjects scoring high on a measure of delusional thinking do show an increased tendency to make false positives, but not to make false negative memory judgements.
\end{abstract}

(c) 2005 Elsevier Ltd. All rights reserved.

Keywords: Schizophrenia; Delusions; Recall; Recognition; False memory; Normal subjects

\footnotetext{
${ }^{*}$ Corresponding author. Fax: +44 1158486826.

E-mail address: keith.laws@ntu.ac.uk (K.R. Laws).
} 


\section{ARTICLE IN PRESS}

\section{Introduction}

Little doubt remains that patients with schizophrenia have substantial memory deficits and that multiple aspects of memory are affected (see Aleman, Hijman, De Haan, \& Kahn, 1999 for a meta-analysis); with deficits reported in short-term memory, working memory, semantic memory, episodic memory, recognition memory and recall. Most of this research has concentrated, however, on forgetting (i.e. false negative memories) and little attention has been directed to problems of false remembering (i.e. false positive memories); or the confidence with which individuals hold false memories.

As well as underlying confabulation in neurological patients, memory disorders have been linked to certain psychotic symptoms including delusional thinking (Johnson \& Raye, 1998). Nevertheless, memory deficits per se are clearly insufficient to cause delusional thoughts because otherwise most amnesics would suffer with delusions. Additional factor(s) must therefore be important. One view of delusions is that the proneness to form false beliefs (delusions) may in part reflect overconfidence in incorrect or implausible judgments or a 'jump to conclusions' way of thinking (Garety \& Hemsley, 1994). Evidence for this comes from research documenting that simple memory intrusions are common in patients with schizophrenia. Studies of source monitoring (Moritz \& Woodward, 2002; Moritz, Woodward, \& Ruff, 2003) reveal an enhanced confidence for false memories in schizophrenics compared to healthy controls. Indeed, Moritz and Woodward (2002) showed that $12 \%$ of high confidence responses made by schizophrenics were errors (compared to a figure of 5\% in controls). Moritz et al. (2003) examined the semantic associations provided by schizophrenics to word lists and then asked them to identify each item as old/new and state the degree of confidence in the word and the attribution. Their results suggested that the core cognitive deficit underlying schizophrenia was the failure to distinguish false from true basic contents; with enhanced confidence for false memories and no differences for correct decisions.

Two recent studies have used the Roediger and McDermott (1995) paradigm to examine false positive memory in schizophrenic patients (Elvevåg, Fisher, Weickert, Weinberger, \& Goldberg, 2004; Moritz, Woodward, Cuttler, Whitman, \& Watson, 2004). In the Roediger and McDermott paradigm, participants are presented with a series of words (e.g. hill, climb, valley, summit, top, molehill, peak, plain, glacier, goat, bike, climber, range, steep) that are strong associates of a nonstudied target item (e.g. 'mountain'). Roediger and McDermott (1995) and others (e.g. Deese, 1959) have found that healthy controls often falsely recall or recognise the unseen target word in subsequent testing and furthermore, such false memories are often given with high confidence. Although they found poorer memory in patients, neither Elvevåg et al. (2004) nor Moritz et al. (2004) found greater incidence of false memories in patients than controls. Nonetheless, these studies are confronted with the problem of poor overall memory in patients with schizophrenia and this may have protected the patients from making false positives. Finally, it is important to note that neither study explicitly examined deluded patients and this could well be a critical factor.

In the current study, we used the Roediger and McDermott (1995) paradigm (see also Deese, 1959) to investigate recall, recognition and memory confidence in healthy individuals with high and low delusional ideation as measured by the Peters et al. Delusions Inventory (PDI: Peters, Joseph, \& Garety, 1999) - a measure of delusional ideation developed for use in the normal population. It has been documented, using formal diagnostic interview, that bizarre delusions were 
reported by around $2 \%$ of the general population (Eaton, Romanoski, Anthony, \& Nestadt, 1991). Studies of normal subjects in this manner overcome the problem of poor memory per se and can explicitly examine for any relationship between delusional thinking and a tendency to produce false positive memories. We predict that proneness to delusional thinking will be associated not just with poorer recall memory, but also with an increased tendency to make false positives (especially for Target words i.e. closely related to the list but not in the list itself). Finally, as with the high conviction attached to many delusions in patient samples, we predict that high PDI scorers will attach high confidence to their false positive memories.

\section{Method}

\subsection{Participants}

One hundred and five undergraduates (55 males and 50 females) aged between 18-31 years old $(M=21, \mathrm{SD}=2.3$ years) were tested. None volunteered a previous history of mental illness, alcohol or drug abuse. Participants received research credits required for fulfilment of their degree programme for taking part in the study. The participants were divided into two non-overlapping groups of high and low PDI scorers by dividing them at the PDI median - this resulted in 54 with Low PDI scores $(M=0.69$; $\mathrm{SD}=0.84$; range $0-2)$ and 51 with High PDI scores $(M=6.43$; $\mathrm{SD}=3.13$; range $3-18)$. The mean of the high PDI scorers was slightly lower, but comparable to that reported by Peters et al. (1999) for their healthy sample of $272(M=9.7 ; \mathrm{SD}=6.7)$ and substantially lower than that reported for their deluded patient sample of $20(M=20.7$; SD $=9)$.

\subsection{Materials/apparatus}

\subsubsection{Peters et al. Delusional Inventory (PDI: Peters et al., 1999)}

This questionnaire was used to measure the level of 'delusional ideation' in healthy normal participants. This measure is based on the Present State Examination (Wing, Cooper, \& Sartorius, 1974) and consists of 40 yes-no questions tapping proneness to unusual thoughts (ranging from religious beliefs to classical delusional thinking: for example, Do you ever feel as if you are a robot or zombie without a will of your own? Do you ever feel as if you are being persecuted in some way? Do you ever feel as if there is a special purpose or mission to your life? Do you ever feel as if your insides may be rotting? Do you ever feel as if other people can read your mind?). Peters et al. (1999) have shown that the PDI has good internal consistency (Cronbach $\alpha=.88$ ), test retest reliability ( $n=83$ over $6-12$ months was 0.82$)$ and criterion validity has been established in people belonging to religious cults and psychotic inpatients showing significantly higher scores than normal controls (Peters, Day, McKenna, \& Orbach, 1999; Peters et al., 1999).

\subsubsection{False memory task (Roediger \& McDermott, 1995)}

This was presented on a computer screen using SuperLab ${ }^{\mathrm{TM}}$ software. There are two parts to this task - recalling a set of eight lists individually and then a short recognition task. The subjects were shown a list of 15 words then asked to recall as many as possible in $2.5 \mathrm{~min}$. The next list was then shown, until all eight were completed. Following this, the recognition task took place with 


\section{ARTICLE IN PRESS}

twenty-three words shown one at a time. The subjects reported their confidence rating in each word (whether it was old or new and whether they were sure or not that the word had occurred or not) based on their memory.

The eight lists were taken from Roediger and McDermott (1995). Each list was composed of associates to a critical target word. The eight critical words used were: Anger, Black, Bread, Chair, Cold, Doctor, Mountain, and Needle. These target words had their respective associate words making up the eight lists with 15 items per list. For example, of the list with the critical word Anger, the 15 associate words were: mad, fear, hate, rage, temper, fury, ire, wrath, happy, fight, hatred, mean, calm, emotion and enrage. Thus, none of the critical items occurred in any of the lists. The recall task was scored as follows: Number of Correct Recalls - the number of words that are recalled only from the lists that have been presented to the subjects (out of 120 words in total). Number of Target Intrusions - the number of critical target words that have been recalled (out of 8 words relating to the eight lists presented). Number of Other Intrusions - words that may not be obviously related to the critical target words (or the words given in the lists) or may be idiosyncratic erroneous recalls.

The recognition test consisted of 23 words presented in random order consisting of: eight studied words and 15 non-studied words ( 8 critical i.e. Target words semantically related to the studied words; and 7 unrelated words). Participants also rated each word as to their confidence that it had occurred in the previously presented lists. A four-point rating scale was used: 1 - sure it was new, 2-probably it was new, 3-probably it was old and 4-sure that the item was old (previously studied in the lists). Comparisons were made for the 'sure' ratings for studied items (including words that they were sure were 'old' i.e. correctly accepted and sure were 'new' i.e. false negatives); target (containing words they were sure were new 'correctly rejected' and sure were old 'false positives') and unrelated (containing words considered as new i.e. 'correctly rejected' and considered as old i.e. 'false positives') words Finally following Moritz et al. (2004), we calculated the 'Knowledge Corruption Index' (based on Moritz et al., 2004), which reflects the proportion of high confidence errors as a proportion of all errors.

\section{Results}

\subsection{Recall}

The recall data are presented in Table 1. This shows that compared to Low PDI scorers, those with High PDI scores recalled significantly more Target, and Other words and significantly fewer Correct words. The percentage of false alarms was $8.04 \%$ for the high PDI group and only $1.95 \%$ for the low PDI group.

Table 1

Mean (and standard deviation) recall memory for correct and incorrect items (targets and others)

\begin{tabular}{lccl}
\hline Memory scores & High PDI $(n=54)$ & Low PDI $(n=51)$ & ANOVA \\
\hline Total of correct words & $92.04 \pm 11.48$ & $97.70 \pm 12.99$ & $F_{1,104}=5.57, p<.02$ \\
Target words & $5.57 \pm 2.04$ & $1.19 \pm 1.43$ & $F_{1,104}=163.89, p<.0001$ \\
Other words & $1.86 \pm 2.24$ & $0.72 \pm 1.17$ & $F_{1,104}=10.82, p<.0001$ \\
\hline
\end{tabular}




\subsection{Recognition and knowledge confidence}

Confidence rating - each word in the recognition phase was rated and group comparisons were made for the 'sure' ratings for studied, target and unrelated words (see Table 2).

The 'Knowledge Corruption Index' (KCI) for high PDI subjects was $46 \%$ compared to only $27 \%$ for the low PDI $\left(F_{1,104}=37.52, p<.001\right)$. This means for high PDI subjects almost half of all errors are high confidence errors (as against just over a quarter for low PDI subjects). When the KCI was calculated separately for false negative and false positive errors, the latter did not differ for high and low PDI subjects (4.89\% vs. 3.15\%: $\left.F_{1,104}=2.25, p=.14\right)$; however, a significant difference emerged for false positives $\left(41 \%\right.$ vs. $\left.24 \%: F_{1,104}=35.30, p<.001\right)$.

\section{Discussion}

The current study has shown that normal healthy subjects who score highly on a measure of delusional thinking have significantly poorer memories than those who score low on a measure of delusional thinking (the PDI: Peters et al. Delusional Inventory). In particular, when compared to the high PDI group, the Low PDI group showed significantly better recall (recalling significantly more correct words, fewer incorrect Target, and Other words). This suggests not only that proneness to delusional thinking is associated with poorer recall memory, but that it affects the tendency to make false positives as well (i.e. for Target and other words). The size of the group difference was especially marked for the Target items (i.e. those that were not in the original list but highly related to the list content), where the high PDI recalled more than four times as many as the low PDI scorers. The High and low PDI subjects did not differ in the confidence they attached to the recognition of items that were in the studied lists; however, for distractor items that were not studied, the High PDI subjects gave less confidence in terms of rejecting unseen items and greater confidence in terms of accepting unseen items. This all suggests that high PDI subjects have a tendency to make false positives (and not to make false negatives).

Table 2

Mean number of words that subjects rated as definitely new and definitely old in the recognition task

\begin{tabular}{llll}
\hline Confidence ratings & $\begin{array}{l}\text { High PDI } \\
\text { Mean }[S D]\end{array}$ & $\begin{array}{l}\text { Low PDI } \\
\text { Mean [SD] }\end{array}$ & ANOVA \\
\hline $\begin{array}{l}\text { Studied }(n=8) \\
\text { Sure new 1 (false negatives) }\end{array}$ & $.75[1.04]$ & $.46[0.79]$ & $F_{1,104}=2.47, \mathrm{~ns}$ \\
Sure old 4 (correct acceptance) & $4.82[1.99]$ & $5.00[1.53]$ & $F_{1,104}=0.26, \mathrm{~ns}$ \\
Target $(n=8)$ & & \\
Sure new 1 (correct rejection) & $.94[1.45]$ & $1.70[1.73]$ & $F_{1,104}=5.99, p=.016$ \\
Sure old 4 (false positives) & $5.16[2.01]$ & $3.09[1.74]$ & $F_{1,104}=31.69, p<.0001$ \\
Unrelated $(n=7)$ & & & \\
Sure new 1 (correct rejection) & $3.07[2.11]$ & $4.14[1.80]$ & $F_{1,104}=7.87, p=.006$ \\
Sure old 4 (false positives) & $1.67[2.41]$ & $.50[1.13]$ & $F_{1,104}=10.25, p<.002$ \\
\hline
\end{tabular}




\section{ARTICLE IN PRESS}

The finding that high and low PDI individuals did not differ in their recognition of words that did occur in the list shows that they were attending to the task. It also shows that it is not a generic failure to distinguish true and false per se, but a decision-making problem that leads them to make false positives. High PDI subjects show a bias, not only, to endorse untrue items, but also to do this with significantly greater confidence than low PDI subjects. In other words, they are confident that they are recognising things they had previously seen (even when they did not). This applies to unseen as well as related items and indicates that it does not depend solely upon the activation of related materials e.g. via abnormal semantic activation. In this context, the evidence from priming studies in patients with schizophrenia may be relevant. Most studies have used priming to achieve this, which involves using the level of assistance that a word (the prime) gives to the processing of another word (the target) as a measurement of their proximity within semantic space. For example, the prime-target pairing lemon-sour would be expected to result in faster responding than lemon-bus. Additionally, because concepts are assumed to be associated within a network, activation may spread, not only to directly related concepts, but also from those concepts to concepts further in the memory network. Some studies have utilized this and employed an indirect priming condition whereby, for example, the word lemon activates the related concept sour, which, in turn activates sweet resulting in lemon priming sweet. Studies examining indirect priming in schizophrenics indicate greater indirect priming (see Spitzer, 1997). In other words, the spread of semantic activation tends to go further and faster. Nevertheless, indirect spreading remains to be compared in patients with and without delusions.

How do the findings reported here relate to those of similar studies of patients with schizophrenia? The first notable thing, is that in two recent studies using the same Roediger and McDermott (1995) paradigm, schizophrenics and controls showed no differences in false positive memory (Elvevåg et al., 2004; Moritz et al., 2004). By contrast, we have reported large differences in false positive memory for normal subjects scoring high and low on delusional ideation. What might be the implications of these findings? The first possibility is that high PDI scores do not relate to the general notion of schizophrenia. The previous studies of schizophrenics did not focus specifically on deluded patients and it could be that this is a critical factor and future studies might compare deluded and non-deluded schizophrenics in comparisons. A second point concerns the possibility that the relationship between false positive memory and delusional ideation only exists in the prodromal phase and disappears with full-blown schizophrenia. One possibility relates to the protection afforded from false positive memories in patients with schizophrenia because they have generally poor memory. The level of recall in the current study (for high and low PDI scorers) was $77 \%$ and $82 \%$, respectively; this is substantially higher than for schizophrenic patients who show floor effects (e.g. Elvevåg et al., 2004). In other words, patients with schizophrenia are less prone to false positive memories because they fail to recall anywhere near as much information as healthy subjects. Further research investigating false memories in schizophrenic patients with delusions would help address these questions and potentially address issues relating to the notion that delusional thought is on a continuum ranging from the normal to the abnormal. Additionally, it should be noted that other variables influence the tendency to produce false positive memories, including dissociation and fantasy proneness (Merckelbach, Muris, Horselenberg, \& Stougie, 2000) and future studies should examine the relationship of these variables with the PDI and their potential for mediating the false memory process. 


\section{ARTICLE IN PRESS}

\section{References}

Aleman, A., Hijman, R., De Haan, E. H. F., \& Kahn, R. S. (1999). Memory impairment in schizophrenia: A metaanalysis. American Journal of Psychiatry, 156, 1358-1366.

Deese, J. (1959). On the prediction of occurrence of particular verb intrusions in immediate recall. Journal of Experimental Psychology, 58, 17-22.

Eaton, W. W., Romanoski, A., Anthony, J. C., \& Nestadt, G. (1991). Screening for psychosis in the general population with a self-report interview. Journal of Nervous and Mental Disease, 179, 689-693.

Elvevåg, B., Fisher, J. E., Weickert, T. W., Weinberger, D. R., \& Goldberg, T. E. (2004). Lack of false recognition in schizophrenia: A consequence of poor memory? Neuropsychologia, 42, 546-554.

Garety, P. A., \& Hemsley, D. R. (1994). Delusions: Investigations into the psychology of delusional reasoning. Oxford: Oxford University Press.

Johnson, M. K., \& Raye, C. L. (1998). False memories and confabulation. Trends in Cognitive Neurosciences, 2, 137-145.

Merckelbach, H., Muris, P., Horselenberg, R., \& Stougie, S. (2000). Dissociative experiences, response bias, and fantasy proneness in college students. Personality and Individual Differences, 28, 49-58.

Moritz, S., \& Woodward, T. S. (2002). Memory confidence and false memories in schizophrenia. Journal of Nervous and Mental Disease, 190, 641-643.

Moritz, S., Woodward, T. S., Cuttler, C., Whitman, C., \& Watson, J. M. (2004). False memories in schizophrenia. Neuropsychology, 18, 276-283.

Moritz, S., Woodward, T. S., \& Ruff, C. C. (2003). Source monitoring and memory confidence in schizophrenia. Psychological Medicine, 33, 131-139.

Peters, E. R., Day, S., McKenna, J., \& Orbach, G. (1999). The incidence of delusional ideation in religious and psychotic populations. British Journal of Clinical Psychology, 38, 83-96.

Peters, E. R., Joseph, S. A., \& Garety, P. A. (1999). Measurement of delusional ideation in the normal population: Introducing the PDI (Peters et al. Delusions Inventory). Schizophrenia Bulletin, 25, 553-577.

Roediger, H. L., III, \& McDermott, K. B. (1995). Creating false memories: Remembering words not presented in lists. Journal of Experimental Psychology: Learning, Memory and Cognition, 21, 803-814.

Spitzer, M. (1997). A cognitive neuroscience view of schizophrenic thought disorder. Schizophrenia Bulletin, $23,29-50$.

Wing, J. K., Cooper, J. E., \& Sartorius, N. (1974). Measurement and classification of psychiatric symptoms: An instruction manual for the PSE and CATEGO program. New York: Cambridge University Press. 